\title{
Percepciones y creencias en educación básica sobre la formación del profesorado en las escuelas normales
}

\section{Perceptions and beliefs in basic education about teacher training in teacher training colleges}

\author{
DOI: $10.46932 / \mathrm{sfjdv2n4-091}$
}

Received in: March 1st, 2021

Accepted in: May 30th, 2021

\author{
Gamboa Robles Marco Antonio \\ Dr. En Planeación y Liderazgo Educativo \\ Escuela Normal Estatal de Especialización \\ Callle Colosio, s/n. Providencia, Sonora, México \\ E-mail: enee.mgamboa@gmail.com \\ María Julieta Maldonado Figueroa \\ Dra. En Educación \\ Escuela Normal Estatal de Especialización \\ Callle Colosio, s/n. Providencia, Sonora, México \\ E-mail: mjmalfig@gmail.com \\ María Angélica Quiroz Leyva \\ Mtra. En Educación Especial \\ Escuela Normal Estatal de Especialización \\ Callle Colosio, s/n. Providencia, Sonora, México \\ E-mail: quirozleyva@gmail.com
}

\section{RESUMEN}

En este documento se presenta a manera de descripción, la interpretación que los docentes en servicio tienen sobre la formación inicial de docentes, particularmente opinan sobre la percepción que tienen del grado de apropiación del perfil profesional que el normalista posee para egresar de la licenciatura en educación especial, como rasgos deseables de competencia docente; con ello se evalúa el conocimiento, habilidades, destrezas, actitudes y valores que los normalistas aplican en sus jornadas de práctica en condiciones reales de trabajo y sus estrategias de atención como parte de la intervención educativa para sujetos que presentan barreras para el aprendizaje y la participación y/o discapacidad en enfoque inclusivo. Considerando que la atención a esta población se ha brindado históricamente de forma diferenciada y con distintos fines y enfoques; desde una mirada asistencial o de corte clínico-terapéutico, hacia una pedagogía incluyente alternativa. Por ello el licenciado en educación especial y en educación inclusiva, constituyen el eje central para llevar a cabo la metodología de aula con perspectiva constructivista competencial en atención a la diversidad, orientada a lograr y fortalecer la igualdad y equidad para facilitar la inclusión e incorporación a la vida social, trabajo productivo y demás ámbitos de la vida cotidiana.

Palabras clave: Competencia docente, práctica educativa, enseñanza situada, aprendizaje significativo.

\section{ABSTRACT}

This document presents, by way of description, the interpretation that in-service teachers have about initial teacher training, particularly their perception of the degree of appropriation of the professional profile that the teacher educator has to graduate from the degree in special education, as desirable traits of teaching 
competence; This evaluates the knowledge, skills, abilities, skills, attitudes and values that the teachers apply in their practice days in real working conditions and their care strategies as part of the educational intervention for subjects who present barriers to learning and participation and/or disability in an inclusive approach. Considering that the attention to this population has historically been provided in a differentiated way and with different purposes and approaches; from a welfare or clinical-therapeutic approach, towards an alternative inclusive pedagogy. Therefore, the graduate in special education and inclusive education, are the central axis to carry out the classroom methodology with a constructivist competence perspective in attention to diversity, aimed at achieving and strengthening equality and equity to facilitate inclusion and incorporation into social life, productive work and other areas of daily life.

Key words: Teaching competence, educational practice, situated teaching, meaningful learning.

\section{INTRODUCCIÓN}

La formación inicial de docentes en educación y las prácticas inclusivas que se desarrollan en los programas de estudio vinculados con el Plan 2004 de la licenciatura en educación especial y el Plan 2018 de la licenciatura en intervención educativa, son caracterizadas por sus diseñadores como fortalecedoras de una visión académica de amplia proyección conceptual y operativa con la finalidad esencialmente formativa sustentado en bases científicas, psicopedagógicas, sociales, filosóficas, epistemológicas, culturales y tecnológicas que propician la formación de nuevos docentes en enfoque competencial, que asume nuevos retos a los profesores y centra las prácticas áulicas en el aprendizaje, con metodologías alternativas de enfoque constructivista para coadyuvar en la resolución de problemáticas en el contexto donde los protagonistas interactúan cotidianamente.

Sin embargo, no hay que perder de vista que, poner en operación un plan curricular basado en competencias, no es un intento nuevo en la formación docente, a pesar de ello y aunque algunos planes de estudio anteriores contemplan este enfoque y puntualizan en roles de docentes y alumnos en el paradigma constructivista y en estrategias centradas en el aprendizaje, hay que ponderar que en el diseño curricular ni el nivel sintético ni analítico basta para obtener éxito, si las prácticas educativas no son congruentes con el modelo; por ello en el marco de los nuevos planes 2018 en las escuelas normales para formar docentes para educación básica, es prioritario movilizar las prácticas docentes y las metodología pedagógicas para que en esta ocasión tengan mayor eficiencia.

En ese sentido es pertinente repensar concienzudamente las prácticas educativas desde varios puntos de énfasis y tomar decisiones de cambio hacia la mejora de nuestra función docente, sobre los cuales se realizan algunas reflexiones para otorgar mayor fundamentación a las prácticas educativas y resignificar lo que las instituciones formadoras de docentes hacemos en la formación del profesorado del siglo XXI; en sintonía con los perfiles de egreso de los normalistas, los principios pedagógicos ideales de un modelo educativo y los parámetros e indicadores del desempeño de los docentes en servicio. 


\section{PLANTEAMIENTO DEL PROBLEMA}

Definir un currículo en competencias requiere del diseño previo de un modelo educativo que considere muy congruentemente el tipo de sujeto que se quiere formar con la operación de planes de estudio y la relación que éste debe ejercer en el contexto donde se desenvuelve; derivado de ello, un modelo pedagógico puede orientar el logro de determinado perfil del egresado a través del enfoque por competencias que considere las condiciones de enseñanza y aprendizaje como situaciones favorecedoras para el desarrollo integral del egresado y su desempeño en los ámbitos de acción, demostrando su capacidad para solventar necesidades involucrando los conocimientos, procedimientos, actitudes y valores necesarios para soluciones eficientes.

En ese sentido, el modelo educativo actual para educación básica y el modelo propuesto para la formación de docentes del siglo XXI, como lo sugiere Marin, (2003) "se fundamenta en la teoría de la educación basada en competencias desde un enfoque holístico que enfatiza en el desarrollo constructivo de habilidades, conocimientos y actitudes que permitan a los y las estudiantes insertarse adecuadamente en la estructura laboral y adaptarse a los cambio y reclamos sociales”. Con ello se pretende la formación de sujetos integralmente desarrollados, que muestren desempeños competentes y pertinentes con la problemática social y productiva para que promuevan el desarrollo de la sociedad; por lo que se sustenta en los cuatro pilares para la educación que propone Delors (UNESCO, 1997): aprender a conocer, aprender a hacer, aprender a convivir y aprender a ser.

El componente pedagógico en este modelo considera un diseño que de acuerdo con González (2000), "enfatiza en una práctica educativa centrada en el aprendizaje, la cual trasciende de la docencia centrada en el estudiante y en la enseñanza. El papel del estudiante y del docente cobra un nuevo sentido. El estudiante construye el aprendizaje a través de la interacción con la información; asumiendo una actitud crítica, creativa y reflexiva que le permite ir aplicando lo que aprende en los problemas cotidianos; por lo que se le considera un sujeto autónomo autogestor de su propio aprendizaje".

Por ello el profesional de la educación que forma formadores, está obligado a vivir su propio proceso formativo acorde al enfoque por competencias donde complete un perfil del egresado de una carrera profesional docente que refleje formación integral de sus competencias profesionales.

La Secretaría de Educación Pública (SEP), órgano rector de la educación en México, ha buscado promover en las últimas tres décadas, una formación docente con énfasis en la preparación para la implementación de un currículo basado en competencias, el cual es una de las tendencias educativas motivadas por las recomendaciones de los organismos internacionales, entre ellos la Organización para la Cooperación y el Desarrollo Económico (OCDE) y la Organización de las Naciones Unidas para la Educación, la Ciencia y la Cultura (UNESCO) (Moreno, 2014). 
Belmonte y Bernárdez (2020), en su estudio, resaltan como principio fundamental, la competencia profesional para la obtención de los títulos de grado en el docente. La finalidad radica en una formación inicial perfilada a los saberes implícitos en la disciplina de la educación; relacionada con el liderazgo, desarrollo y evolución, presentes en la vida educativa y en todos sus ámbitos. El fomento del pensamiento crítico y la inteligencia práctica, son los elementos esenciales para la adquisición de aprendizajes permanentes, según el modelo de formación en competencias en el que respaldan el perfil profesional.

La problemática compleja en este sentido, no sólo se centra en torno al logro del perfil de egreso, es relevante cuidar el perfil de ingreso; pero no el perfil de ingreso de los aspirantes a cursar las carreras de docencia, sino, el perfil de ingreso de los docentes que se contratan en las escuelas normales, pues en innumerables situaciones no cuentan con las competencias ni el carácter para ser formadores, porque se alejan de los enfoques modernos y de la pedagogía constructivista que diseñe los escenarios de aprendizaje donde se creen ambientes de aprendizaje propios del nuevo modelo educativo.

Con base en lo anterior el presente estudio busca responder a la pregunta:

¿Cuál es la percepción de los docentes en servicio con relación al logro del perfil de egreso de los futuros docentes de educación especial egresados de la Escuela Normal Estatal de Especialización?

Esta interrogante se plantea con el propósito de vincular la temática general de esta investigación cuyo enlace se establece directamente con las funciones y responsabilidades de los normalistas en su desempeño profesional entorno a la intervención educativa que ponen en práctica en las jornadas de trabajo docente; concebir que esta práctica tiene como campo de acción la educación básica y, que su desempeño profesional es un factor fundamental para cubrir una preciada meta en nuestro sistema educativo para formar integralmente a los ciudadanos que queremos formar, lo cual se logra en coordinación con el compromiso y esfuerzo de los actores implicados en el proceso formativo, como son: alumnos, maestros, directivos y autoridades educativas.

El reto que se desprende de un análisis de práctica docente es fortalecer la formación inicial del profesorado, sabiendo que es un proceso complejo y, su puesta en marcha implica dificultades, pero es sin duda un desafío que debe atenderse prioritariamente en las escuelas normales, pues se trata de fortalecer y transformar a las instituciones que preparan a los maestros que México requiere porque realizan una aportación insustituible al mejoramiento de la educación del país.

Objetivo general

Caracterizar la opinión externa sobre el desempeño de los estudiantes normalistas, como mecanismo de evaluación para la toma de decisiones que orienten la transformación de prácticas docentes en la formación del profesorado en la Escuela Normal que responda a la atención a la diversidad en contextos inclusivos. 


\section{MARCO TEÓRICO}

\subsection{IMPLICACIONES CONTEMPORÁNEAS EN LA FORMACIÓN DOCENTE}

Focalizar particularmente los modelos de enseñanza en la formación de docentes que se realiza en las escuelas normales en México, ha sido un reto que ha mantenido preocupados y ocupados a las autoridades correspondientes en la subsecretaria de educación superior de la SEP, desde 1984 cuando la formación de docentes se posicionó en el rango de licenciatura universitaria, se realizaron diseños curriculares para buscar la formación de profesionales de la educación que posibilitara la educación de los estudiantes como sujetos activos, capaces de impactar en la mejora del mundo desde el enfoque cognitivista, lo que dejó muchas limitaciones para responder con mejor calidad al contexto social. Sin embargo en 1997 se inicia un programa de transformación para formar nuevos licenciados en educación para el nivel básico, dicho programa iniciaba el cambio de paradigma para transformar la formación de docentes centrada en el constructivismo, con prácticas docentes centradas más en el aprendizaje que en la enseñanza, dado que en educación básica ese paradigma había cambiado desde 1993, por lo que había que formar profesores capaces de enfrentar las nuevas realidades.

Particularmente desde inicios del siglo XXI la formación docente ha asumido el enfoque constructivista, el cual se sitúa en la dimensión que considera la perspectiva hermenéutica para interpretar el mundo en que se vive y en construcción colaborativa social transformar el contexto dinámico resolviendo la problemática que permita atender las necesidades del sujeto y de su núcleo social; en este enfoque, la enseñanza consiste en crear las condiciones adecuadas de autodescubrimiento personal, por lo que la formación del profesorado adquiere dimensiones personales, relacionales, situacionales e institucionales que deben ser considerados para facilitar a cada sujeto su desarrollo personal acorde con Combs (1979), cuando afirma que "El profesor eficaz es un ser humano único que ha aprendido a hacer uso de sí mismo eficazmente, y a llevar a cabo sus propios propósitos y los de la sociedad en la educación de otras personas".

En 2012, el modelo de formación de docentes para educación primaria y preescolar, se redefine para agregar al enfoque constructivista, el modelo por competencias y el foco del aprendizaje centrado en el alumno. Después de un largo período de análisis curricular, se concretiza el rediseño del modelo en la formación docente para todos los niveles de educación obligatoria, puntualizando lo que se decidió en 2012: posicionar el constructivismo, hacer énfasis en el modelo por competencias, centrar el proceso en el aprendizaje del alumno, optimizar el uso de las TIC y cubrir los estándares de calidad y equidad educativa en ambientes inclusivos, iniciando simultáneamente la operación de nuevos planes en 2018, a la par del nuevo modelo en educación obligatoria. 
Los nuevos diseños curriculares para las 16 licenciaturas que entran en operación en agosto de 2018, coordinados por la DGESPE, se sustentan en las teorías de los modelos de enseñanza que por sus fines corresponden a diferentes familias de acuerdo a la caracterización de Joyce y Weil (2000), dado que se encuentran aportaciones teóricas tanto del modelo del estudiante como centro de enseñanza, como del modelaje de estados fructíferos del crecimiento y de los centrados en la investigación para el desarrollo y autorrealización de la familia de modelos personales. Igualmente se retoman aportes relevantes de la familia de los modelos sociales, particularmente de la cooperación entre pares, el reconocimiento de los valores de la sociedad y el de adaptación a las diferencias individuales. Otras aportaciones teóricas se refieren a modelos sobre la inclusión que atienden a la equidad o la consideración de condiciones de aprendizaje y lo relativo a enseñar a aprender.

Derivado de dicho modelo se orientan los diseños de prácticas para realizar la intervención educativa que atienda la nueva formación inicial de docentes, la cual se puede analizar desde los seis criterios de comparación de Reigeluth (1999), ya que por su intencionalidad el diseño curricular 2018 atiende tanto al desarrollo social como al desarrollo emocional; mientras que por enfoque teórico encuentra sustento en el paradigma constructivista y en el enfoque interdisciplinario para atender el aprendizaje basado en problemas o proyectos; por su parte la flexibilidad que se percibe en el diseño es alta, puesto que es un eje importante como elemento del mismo modelo tanto en lo académico como en lo administrativo; por su nivel de aplicabilidad se encuentra énfasis en el nivel de estructuración bajo permitiendo concretar los nivel meso y micro del currículo sin problema, con un foco de aprendizaje amplio, y un andamiaje de bajo control lo que genera mayor autonomía al alumno; mientras que por el criterio de configuración se posibilitan todas las modalidades de agrupamiento para aprender y se priorizan las relaciones alumno-alumno y alumno-ambiente para la construcción de aprendizaje; por su parte las condiciones docentes requieren de docentes expertos para propiciar la construcción de aprendizaje, expertos en las metodologías pedagógicas y las competencias profesionales para provocar el aprender a aprender, aprender a hacer y aprender a ser.

\subsection{EL ENFOQUE COMPETENCIAL EN LA FORMACIÓN DOCENTE DEL CONTEXTO MUNDIAL}

El sistema educativo actual, moviliza tres elementos esenciales que se relacionan entre sí, como un sistema coherente para el profesionalismo docente; siendo éstas, las competencias, la formación y la evaluación. Desde la perspectiva de este modelo educativo, la calidad de la enseñanza, para promover altos niveles de aprendizaje en los estudiantes, sigue siendo el reto que vendrá a constituir una buena docencia, y por consiguiente, la garantía de una profesión sólida. 
Las exigencias actuales en la formación de formadores, resalta la competencia docente como eje transformador en el quehacer de este profesional. Cultivar y conservar esta práctica, requiere tanto construcción como fortalecimiento a lo largo de su trayectoria, es decir, desde la inducción a la profesión, el desarrollo profesional continuo, y la evaluación de la práctica. La iniciativa de partida recae en la comprensión íntegra de los estándares docentes que potencialicen la formación de los educadores.

En este sentido, la competencia docente involucra directamente la calidad de la enseñanza, con acciones que requieren de una profunda comprensión de los contenidos, un exhaustivo conocimiento de los estudiantes y un amplio repertorio de estrategias pedagógicas. Así mismo, desafía el fortalecimiento de la profesión, al exigir altos niveles de aprendizaje de los estudiantes, y por lo tanto, también altos niveles de habilidades en el formador.

La evaluación de la competencia docente, es medida bajo políticas de formación profesional basadas en estándares de práctica. La docencia por lo tanto, al considerarse un conjunto de habilidades y conocimientos cuantificables y medibles, puede ser aprendida y desempeñada con eficacia, cuando hay una motivación intrínseca incluso extrínseca en el individuo (Zabalza y Veraza, 2003).

En el caso de la formación docente de las Escuelas Normales (EN), los estándares de medición que la evalúa, están especificados en los rasgos del perfil de egreso de la disciplina en la que se forma el normalista. Por lo tanto, el objetivo de aplicar una buena docencia, se traduce al compromiso que tiene el formador, de desarrollar competencias docentes en el futuro profesional de la educación y garantizar que todos los egresados de sus programas, hayan adquirido los conocimientos y habilidades descritos en los estándares curriculares que los rigen.

En este sentido, ante la exigencia actual en la formación de formadores, se vuelve indispensable contar con un marco referente sobre posibles líneas de acción y mejoramiento de la práctica pedagógica, tal y como lo hacen algunos países considerados del tercer mundo en la educación, estos son Estados Unidos, Autralia, Reino Unido, Cuba y Chile (Bayona-Rodríguez, Urrego y Hernández, 2017).

Danielson (2011), propone un marco para la enseñanza y el aprendizaje basado en una visión constructivista. Su estudio determina cuatro dominios de la responsabilidad docente, cada uno con diversos mecanismos de instrucción que en conjunto generan 22 componentes de la enseñanza. En este sentido, según el autor, todo formador debe contemplar como parte de sus competencias, la planificación y preparación, el ambiente en el aula, la enseñanza y las responsabilidades profesionales.

El marco de desarrollo y desempeño docente promueve la excelencia de la enseñanza y el liderazgo escolar en Australia desde el año 2008. Se implementa un plan de estudios nacional, en el que se establecen los estándares para profesores y directores. Aquí, se resalta la conectividad tecnológica a través de siete plataformas políticas para la promoción de excelencia docente, estableciendo como principales categorías 
los conocimientos, habilidades prácticas, compromiso profesional de los docentes, estructura para la valoración, desarrollo, mejora de la práctica docente, retroalimentación. Así mismo, promover la práctica profesional profunda reflexiva y colaborativa (James, 2008).

Se visualiza así, la importancia de fortalecer la formación inicial de docentes, que para el caso de México, Matthews et al., (2010) sostienen en OCDE (2010) que "Las Normales públicas y privadas y otras instituciones de formación inicial docente necesitan mejorar sustancialmente si pretenden ser el principal medio del país para preparar a sus docentes. El primer paso debe ser establecer un sistema de estándares rigurosos para acreditar a todas las Normales y demás instituciones de formación inicial” ( $p$, 13).

En el caso de Cuba, se cuenta con un marco de desempeño docente, que exige estándares, organizados en dimensiones e indicadores; la evaluación de los docentes busca

“Conocer de forma integral las características personales y la preparación político - ideológica, científica, pedagógico - metodológica de los profesores, maestros, educadoras, instructores de enseñanza práctica, bibliotecarios escolares, auxiliar de reeducación, auxiliar técnico docente, psicopedagogo, pedagogo clínico, psicólogo y logopeda y mantener una constante atención sobre su desarrollo, de modo que la evaluación cumpla su función educativa; verificando: 1. resultados del trabajo, 2. preparación para el desarrollo del trabajo y 3. características personales y cumplimiento de las normas de conducta y principios de la Ética Pedagógica y Profesional" (Valdés, 2013, p, 8).

En chile, manejan un sistema nacional de desempeño docente, implementado por maestros, el sindicato y el gobierno; conocido como marco para la buena enseñanza (MBE). Este maneja cuatro dominios docentes, que refieren la planeación y preparación de la enseñanza, la creación de ambientes propicios para el aprendizaje, la enseñanza para el aprendizaje de todos los estudiantes y las responsabilidades profesionales. El instrumento incorpora las responsabilidades de los docentes en su quehacer cotidiano, orienta a la auto reflexión, crítica y evaluación del desempeño y promueve potenciar el desarrollo profesional a través de la calidad educativa brindada (CPEIP, 2008).

Las anteriores investigaciones internacionales, aun manifestadas en culturas muy diferentes, dejan entrever la coincidencia de elementos necesarios e indispensables a tener en cuenta para la actuación profesional de la formación docente, que legitime y oriente su intervención educativa en todas las vertientes. Por consiguiente, los estándares curriculares que rigen la formación docente, se traducen en la coyuntura de la profesión de formadores, que resulta relevante se lleve a discusión y determinación del marco de enseñanza docente que requiere nuestro país. 


\section{METODOLOGÍA}

\subsection{TIPO DE ESTUDIO}

El presente trabajo es un estudio con un enfoque cuantitativo de diseño no experimental de tipo transversal descriptivo. Según Hernández, Fernández y Baptista (2010), los diseños no experimentales son investigaciones sistemáticas y empíricas en la que las variables independientes no se manipulan porque ya han sucedido. Las inferencias sobre las relaciones entre variables se realizan sin intervención o influencia directa y dichas relaciones se observan tal y como se han dado en su contexto natural. Estos mismos autores indican que los estudios transversales descriptivos recolectan datos en un solo momento, en un tiempo Único. Su propósito es describir variables, y analizar su incidencia e interrelación en un momento dado. En este caso, el fin principal es conocer la percepción que tiene los docentes en ejercicio profesional, sobre el nivel de logro del perfil de egreso que los normalistas presentan en la última etapa de su formación inicial en la licenciatura.

\subsection{PARTICIPANTES}

Con base en el objetivo del estudio, se realizó un muestro de tipo intencional puesto que se buscaba seleccionar una población con características muy definidas, en este sentido, se incluyeron a todos tutores de los alumnos de último grado de la Licenciatura en Educación Especial de la Escuela Normal Estatal de Especialización del Estado de Sonora. La población se constituyó por 150 sujetos que en el momento del estudio se encontraban cubriendo funciones de tutores en las prácticas educativas de los alumnos de la generación 2014-2018; con quienes realizaban funciones de asesoría y acompañamiento con el fin de identificar buenas prácticas y propiciar una intervención educativa congruente con el modelo educativo que se implementa en educación básica a partir del 2018.

\subsection{PROCEDIMIENTO}

En la última semana de trabajo docente, que los normalistas realizan por más de 40 semanas, como práctica profesional para culminar su carrera, se aplicó un instrumento a los docentes en ejercicio profesional que los acompañaron y evaluaron durante dicho período de práctica, la aplicación es instrumentada técnicamente por el equipo de investigadores que pertenecen a un Cuerpo Académico En Formación de la Escuela Normal Estatal de Especialización. Debido a aspectos de carácter ético, la aplicación de instrumento se llevó a cabo con la participación consentida de cada sujeto, la participación se efectuó de manera presencial en grupos de 10 a 15 personas para explicar los fines del estudio previamente. 
Para el análisis estadístico se utilizó el programa SPSS, para la obtención de análisis de frecuencias, además de realizarse una prueba paramétrica para una sola muestra de t de Student, con el mismo software como herramienta y teniendo como referencia la media teórica de 3.

La prueba t de Student para una muestra permite comprobar si es posible aceptar que la media de la población es un valor determinado. Se toma una muestra y la prueba permite evaluar si es razonable mantener la Hipótesis nula de que la media es tal valor.

Cabe mencionar que casi todos los reactivos siguen una distribución positiva, lo que quiere decir que los puntajes mayores a 3 representan una característica positiva respecto a la pregunta.

De igual forma, se llevó a cabo un análisis factorial confirmatorio con la técnica de componentes principales y rotación varimax pues en todos los casos se suponía que las dimensiones de cada una de las escalas del instrumento eran ortogonales, se obtuvieron los pesos factoriales y se estuvo en condición de decidir la mejor configuración factorial asociada a la teoría. Aquellos reactivos que no alcanzaron un peso factorial de 0.400 en la solución elegida, fueron dados de baja. Los reactivos que permanecieron dentro de las dimensiones de la Escala FIT fueron analizados a través del estadístico alfa de Cronbach, con el fin de observar su consistencia interna y decidir sobre la importancia relativa de cada uno de ellos.

\section{DESARROLLO Y DISCUSIÓN DE RESULTADOS}

A continuación se presenta en la tabla 1, los principales resultados de las medias de los diferentes reactivos que conforman el instrumento de medida, así como el rasgo de perfil de egreso vinculado a los reactivos, cabe destacar que este primer momento se realiza un análisis por cada reactivo que conformaba el instrumento, asociado a un indicador del perfil de egreso.

Tabla 1. Rasgos del perfil de egreso por campos

\begin{tabular}{|c|c|c|c|}
\hline Rasgo del perfil & Reactivo & Media & Desviación estándar \\
\hline \multirow{4}{*}{$\begin{array}{l}\text { Habilidades } \\
\text { intelectuales } \\
\text { específicas }\end{array}$} & Expresa sus ideas con claridad y sencillez & 3.61 & .49 \\
\hline & $\begin{array}{l}\text { Plantea, analiza y resuelve problemas y desafíos intelectuales en su práctica } \\
\text { profesional para sus alumnos }\end{array}$ & 3.56 & .49 \\
\hline & $\begin{array}{l}\text { Realiza reflexión crítica sobre su práctica docente para mejorar su labor } \\
\text { educativa }\end{array}$ & 3.55 & .50 \\
\hline & $\begin{array}{l}\text { Muestra habilidad e iniciativa para continuar aprendiendo y enriquecer su } \\
\text { práctica docente }\end{array}$ & 3.66 & .47 \\
\hline \multirow{5}{*}{$\begin{array}{l}\text { Conocimiento } \\
\text { los propósitos }\end{array}$} & Conoce propósitos y contenidos de educación básica & 3.53 & .50 \\
\hline & Tiene claridad de la misión de educación especial & 3.75 & .43 \\
\hline & $\begin{array}{l}\text { Reconoce la secuencia lógica de los contenidos de las asignaturas y es capaz } \\
\text { de articularlos }\end{array}$ & 3.58 & .49 \\
\hline & $\begin{array}{l}\text { Establece correspondencia adecuada de los propósitos y contenidos, con los } \\
\text { procesos desarrollo con la diversidad de capacidades de estilos y ritmos de } \\
\text { aprendizaje y el contexto social y familiar de sus alumnos }\end{array}$ & 3.66 & .47 \\
\hline & $\begin{array}{l}\text { Domina los fundamentos, principios y finalidades de la educación especial, } \\
\text { lo relaciona con educación básica }\end{array}$ & 3.62 & .48 \\
\hline
\end{tabular}




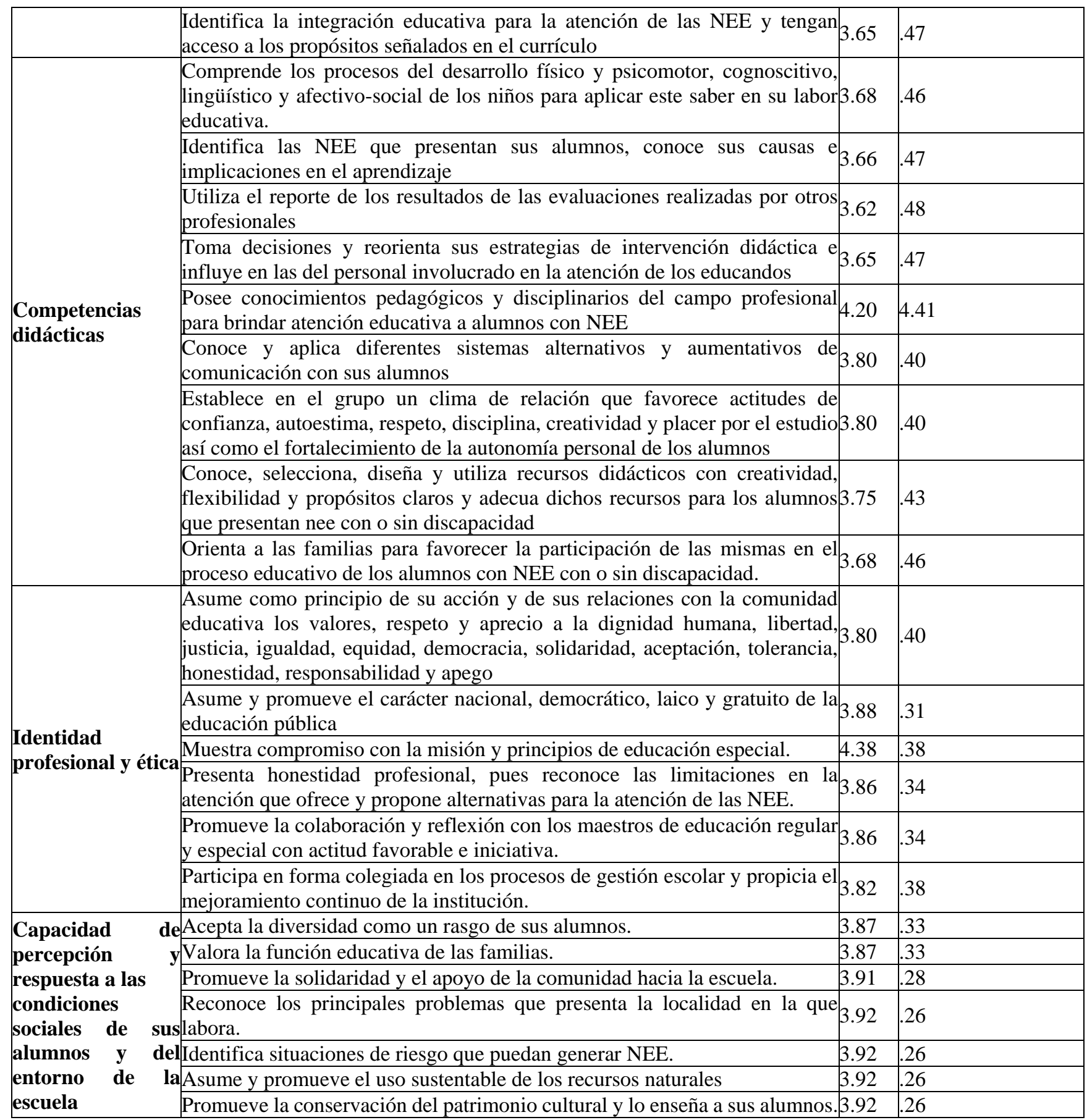

En la primera parte del análisis se buscó revisar las medias de los diferentes reactivos que conformar las dimensiones relacionadas con el perfil de egreso, en todos los casos los reactivos obtuvieron valores por encima del punto de corte medio (2.5), lo cual hace referencia a una percepción positiva de los diferentes rasgos de los alumnos.

Sin embargo, debido a la distribución anormal que presenta la curva de la población (cargada hacia la derecha) y el tamaño de la población $(\mathrm{N}=150)$ no fue posible realizar análisis paramétrico de las observaciones, por lo cual, se recurrió a realizar análisis no paramétrico con la finalidad de explorar las 
relaciones entre las variables del estudio; considerando en un segundo momento los campos o categorías que conforman el perfil deseable del egresado de licenciatura en educación.

Tabla 2. Correlaciones entre campos de perfil de egreso

\begin{tabular}{|c|c|c|c|c|c|c|}
\hline & & $\mathrm{HI}$ & $\mathrm{CP}$ & $\mathrm{CD}$ & ID & CPRC \\
\hline \multirow[t]{2}{*}{ Habilidades intelectuales específicas } & $\begin{array}{l}\text { Correlación } \\
\text { Pearson }\end{array}$ & & .54 & $.244^{*}$ & -.026 & .023 \\
\hline & Sig. (bilateral) & & .000 & .029 & .819 & .841 \\
\hline \multirow[t]{2}{*}{ Conocimiento de los propósitos } & $\begin{array}{l}\text { Correlación } \\
\text { Pearson }\end{array}$ & $\mathrm{de} .542^{* *}$ & & $.336^{* *}$ & -.037 & $.290^{* *}$ \\
\hline & Sig. (bilateral) & .000 & & .002 & .744 & .009 \\
\hline \multirow[t]{2}{*}{ Competencias didácticas } & $\begin{array}{l}\text { Correlación } \\
\text { Pearson }\end{array}$ & $\mathrm{de} .244^{*}$ & $.336^{* *}$ & & .073 & $.302^{* *}$ \\
\hline & Sig. (bilateral) & .029 & .002 & & .518 & .006 \\
\hline \multirow[t]{2}{*}{ Identidad profesional y ética } & $\begin{array}{l}\text { Correlación } \\
\text { Pearson }\end{array}$ & & -.037 & .073 & & \\
\hline & Sig. (bilateral) & .819 & .744 & .518 & & .452 \\
\hline $\begin{array}{l}\text { Capacidad de percepción y respuesta a las } \\
\text { condiciones sociales de sus alumnos y de }\end{array}$ & $\begin{array}{l}\text { Correlación } \\
\text { Pearson }\end{array}$ & $\mathrm{de}_{.023}$ & $.290^{* * *}$ & $.302^{* *}$ & -.085 & \\
\hline entorno de la escuela & Sig. (bilateral) & .841 & .009 & .006 & .452 & \\
\hline
\end{tabular}

**. La correlación es significativa en el nivel 0,01 (2 colas).

*. La correlación es significativa en el nivel 0,05 (2 colas).

Para analizar las relaciones entre las variables se sometió a los datos a un análisis de correlación de Kendall, este análisis arrojó correlaciones significativas para casi todas las dimensiones del perfil, para la variable de habilidades intelectuales específicas esta resulto significativa con las variables de conocimiento de los propósitos y competencias didácticas, sin embargo no fue así para la variable de capacidad de percepción y respuesta a las condiciones sociales de sus alumnos y del entorno de la escuela, esto último hace notar una desvinculación entre los conocimiento y la praxis en el contexto de la escuela. Por otro lado, la variable de conocimiento de los propósitos resultó significativa para todas las demás variables lo cual señala la importancia del conocimiento de los programas para tutores, por último, es importante señalar como la variable de identidad no resultó con ninguna correlación significativa con las demás variables, esto puede deberse a que la población percibe a sus tutorados con una identidad docente poco relacionada con la presentada en el perfil de egreso.

\section{CONCLUSIONES}

Primeramente con relación a la percepción de los tutores sobre las competencias de los egresados sobre su perfil, se puede considerar que estos tienen una percepción positiva de los mismos por encima del punto de corte, sin embargo, estos no presentan un puntaje elevado cercano al 5 en ninguno con excepción del rasgo posee conocimientos pedagógicos y disciplinarios comunes del campo profesional para brindar atención educativa a alumnos con NEE, lo cual es congruente con la formación de los 
alumnos, puesto que son formados en educación especial, sin embargo la percepción de desempeño aún no es la mejor posible.

Por otro lado, dentro del análisis de correlación las dimensiones del perfil de egreso, se hace presente la desvinculación del rasgo de identidad profesional y ética con relación a los de habilidades intelectuales específicas, conocimiento de los propósitos, competencias didácticas y capacidad de percepción y respuesta a las condiciones sociales de sus alumnos y del entorno de la escuela. Señala una serie de retos para la formación de los futuros docentes de educación especial, pues desde el punto de vista de los tutores, esta área presenta poco concordancia con los demás, en este sentido, se hace necesario la continuidad en los estudios con este tipo de población que busquen aceptar o refutar la hipótesis de que los egresados de la licenciatura en educación especial no están siendo formados de manera adecuada en el campo de la ética.

Tal como mencionan Mellado (2013) los cambios en la realidad educativa de los últimos años hace necesaria una reflexión de la forma mediante la cual debemos de hacer frente a dichos cambios sin descuidar una filosofía ética.

Con relación a la construcción de la identidad docente, Fuquene y Martínez (2013) mencionan como con relación a la inclusión se puede observar una identidad "parchada" es decir, existe una tendencia a "rellenar" la identidad de los docentes con la necesidad de incluir a los alumnos con NEE dentro de sus grupos.

Esto último podría influir en como los tutores perciben a los docentes supervisados aún sin una identidad clara sobre su propia formación, otro de los factores relevantes dentro de la conformación de estos docentes podría ser la edad, debido a que al presentar un poco práctica la identidad de ellos mismo aún no se encuentra del todo conformado.

Por último, es necesario presentar las limitaciones del estudio para lograr en un futuro mejorar el alcance de los resultados, por lo cual, se hace necesario, ampliar la muestra de estudio, incluir mediciones más precisas y recabar datos que permitan contextualizar las percepciones de los tutores. 


\section{REFERENCIAS}

Bayona-Rodríguez, H., Urrego, L. y Hernández, N. (2017). Marcos para las buenas prácticas de enseñanza. Recuperado de: https://www.magisterio.com.co/articulo/marcos-para-las-buenas-practicasde-ensenanza

Belmonte, María. y Bernárdez, Abraham. (2020). Perfiles profesionales del pedagogo. Florida Journal of Development, Miami, v.1, n.4, p. 305-319, oct./dec. 2020. ISSN 2675-5459. https://doi.org/10.46932/sfjdv1n4-014

Combs, A. y Cols. (1979): Claves para la formación de los profesores. Un enfoque humanístico. Madrid: EMESA.

Danielson, C. (2011). Competencias docentes: desarrollo, apoyo y evaluación (Vol. 20). CINDE.

Fúquene, C. A. A., \& Martínez, Á. G. (2012). Construcción de la identidad profesional docente: ¿Posibilidad o utopía? En Revista EDUCyT, 2012; Vol. Extraordinario, Diciembre, ISSN 2215 - 8227 de la Asociación Colombiana para la investigación en Educación en Ciencias y Tecnología. Bogotá.

James, M. (2008). Aplicación de sistemas para la evaluación docente en escuelas australianas. Melbourne: Instituto Australiano de la Enseñanza y el Liderazgo Escolar.

Joyce, B.; Weil, M., \& Calhoun, E. (2000). Modelos de enseñanza. Barcelona, España: Gedisa, 2000

Mazario Triana, Israel. (2013). Estrategias didácticas para enseñar a aprender. Centro de Estudio y Desarrollo Educacional. Universidad de Matanzas. Cuba.

Mellado, J. A. I. M. (2013). Ética docente del siglo XXI: Nuevos desafíos. Edetania: estudios y propuestas socio-educativas, (43), 17-31.

Moreno-Anguas, M. (2014). Los organismos internacionales y las políticas de formación docente. En Ducoing, P. (Coord.). La escuela normal, una mirada desde el otro. México: Universidad Nacional Autónoma de México. Recuperado de http://132.248.192.241/ editorial/wp-content/uploads/2014/10/LaEscuela-Normal.pdf

OCDE. (2010). Mejorar las escuelas estrategias para la acción en México. Informe de la OCDE sobre el resultado del acuerdo establecido con la Secretaría de Educación Pública (SEP) para mejorar la calidad y la equidad del sistema educativo en México (2008-2010). Recuperado de http://mapeal.cippec.org/wpcontent/uploads/2014/05/OCDE-Mejorar-las-escuelas-estrategias-para-la-acci\%C3\%B3n-en-

M\%C3\%A9xico.pdf

Reigeluth, Charles M. (1999). Diseño de la instrucción. Teorías y modelos. Un nuevo paradigma de la teoría de la instrucción. Tomo II. Buenos Aires, Argentina: Santillana, Aula XXI.

Valdés, Veloz, Héctor. (2013). La evaluación del desempeño del docente: un pilar del sistema de evaluación de la calidad de la educación en Cuba. Instituto de Ciencias Pedagógicas. habana. Recuperado de https://docentemas.cl/docs/La_evaluacion_del_desempeno_del_docente_en_Cuba.pdf

Zabala Vidiella, Antoni y Arnau Belmonte, Laia (2007). 11 ideas clave: Cómo aprender y enseñar competencias. 2da. Edición. Editorial Graó. España. 
Zabalza, M. Á., \& Beraza, M. Á. Z. (2003). Competencias docentes del profesorado universitario: calidad y desarrollo profesional (Vol. 4). Narcea Ediciones. 